(C) Dereito Vol.27, no:107-126 (Xullo-Decembro, 2018) • ISSN 1132-9947

\title{
UNA APROXIMACIÓN ECONÓMICA A LOS FUNDAMENTOS DE LOS DERECHOS INALIENABLES ${ }^{1}$
}

An economic approach to the foundations of inalienable rights

DOI: http://dx.doi.org/10.15304/dereito.27.2.5494

José Miguel BelLo VilLarino

Doctorando en Derecho

Universidad de Sydney

jbel6800@uni.sydney.edu.au

\section{Resumen}

Este artículo es una reacción a la crítica relativista a la existencia de unos derechos universales de carácter inalienable. Si bien este debate se encuentra hoy relativamente amortiguado, ello parece ser debido a un cierto conformismo en el mundo académico y en la práctica internacional con la necesidad de cierta adaptación de los derechos humamos a las culturas locales. Con métodos propios del análisis económico del derecho, pero con un formalismo matemático de mínimos, se deriva la existencia de ciertos derechos inalienables y universales, fundamentados en las preferencias del Homo economicus.

Palabras clave: derechos humanos, análisis económico, relativismo, derechos inalienables, Homo economicus.

\section{Abstract}

This article is a reaction to the relativist critic challenging the existence of universal inalienable rights. Although the debate is relatively muted today, this seems to be due to a certain degree of conformism in academia and among international practitioners regarding the need for a certain adaptation of human rights to local cultures. With a methodology borrowed from economic analysis of the law, but with minimal mathematical formalism, the article sets the foundations for the existence of certain inalienable and universal rights, based on the preferences of the Homo economicus.

Keywords: human rights, economic analysis, relativism, inalienable rights, Homo economicus.

\section{SUMARIO}

1.INTRODUCTION.; -2. GENDER EQUALITY.; -3.PUBLIC PROCUREMENT.; 1.INTRODUCCIÓN.; -2.LA BÚSQUEDA DE UNA NUEVA FUNDAMENTACIÓN

\footnotetext{
1 Una versión preliminar de este artículo fue presentada en la Segunda Conferencia Australiana sobre Teoría Política en Sídney en febrero de 2018. El autor agradece las sugerencias y críticas allí recibidas, así como los comentarios previos de W. Kerber y R. Vijeyarasa. La versión actual se ha beneficiado notablemente de las observaciones de los dos revisores anónimos de esta revista. Los errores, en cambio, son sólo atribuibles a este autor.
}

Recibido: 04/09/2018. Aceptado: 05/12/2018. 
PARA LOS DERECHOS INALIENABLES: ¿POR QUÉ Y PARA QUÉ?.; -3.SIETE PASOS PARA SENTAR LAS BASES ECONÓMICAS DE LOS DERECHOS INALIENABLES; - -3.1.EL SER HUMANO ES UN HOMO ECONOMICUS.; -3.2. EL SER HUMANO NUNCA SE ENFRENTA A UNA DECISIÓN SOBRE QUÉ DERECHOS DEBERÍAN SER SUS DERECHOS INALIENABLES.; -3.3.EL VELO DE LA IGNORANCIA RAWLSIANO ES UNA HERRAMIENTA ÚTIL PARA SOBREPASAR ESA IMPOSIBILIDAD INTRÍNSECA.; -3.4.EL HOMO ECONOMICUS DESEA SUAVIZAR EL CONSUMO; -3.5.EN LA SITUACIÓN DE PREEXISTENCIA, LA ELECCIÓN DE UN CONJUNTO DADO DE REGLAS FUNDAMENTALES DE LA COMUNIDAD PUEDE VERSE COMO UN MERCADO.; -3.6.TODO INDIVIDUO ESTARÍA DE ACUERDO CON ESTAR COMPLETAMENTE ASEGURADO CONTRA ALGUNOS DE LOS RIESGOS.; 3.7.CUANDO CADA INDIVIDUO DESEA ASEGURARSE DE MANERA COMPLETA E INCONDICIONAL, LA NORMA QUE ESTABLECE ESE SEGURO PROTEGE UN DERECHO INALIENABLE.; - 4.OBSERVACIONES A MODO DE CONCLUSIÓN. ; -.5. BIBLIOGRAFÍA.

\section{SUMMARY}

1.INTRODUCTION.; -2.A SEARCH FOR A NEW FOUNDATION FOR INALIENABLE RIGHTS: WHY AND WHAT FOR?.; -3.SEVEN STEPS TO SET THE ECONOMIC BASIS OF INALIENABLE RIGHTS.; -3.1.THE HUMAN BEING IS AN HOMO ECONOMICUS.; -3.2.THE HUMAN BEING NEVER FACES A DECISION ABOUT WHICH RIGHTS SHOULD BE INALIENABLE.; 3.3.THE RAWLSIAN VEIL OF IGNORANCE IS A USEFUL TOOL TO OVERCOME THAT INTRINSIC IMPOSSIBILITY.; 3.4.THE HOMO ECONOMICUS DESIRES TO SMOOTH CONSUMPTION.; -3.5.IN THE PREEXISTENCE SITUATION, THE CHOICE OF A SET OF FUNDAMENTAL RULES FOR THE COMMUNITY COULD BE SEEN AS A MARKET.; -3.6.EVERY INDIVIDUAL WOULD AGREE TO BE COMPLETELY INSURED AGAINST CERTAIN RISKS.; -3.7.WHEN EVERY INDIVIDUAL WANTS COMPLETE AND UNCONDITIONAL INSURANCE, THE NORM THAT ESTABLISHES THAT INSURANCE PROTECTS AN INALIENABLE RIGHT.; -4.SOME OBSERVATIONS AKIN TO A CONCLUSION.;-5.BIBLIOGRAPHY.

\section{INTRODUCCIÓN}

"Derechos naturales", 2 "derechos humanos", 3 "derechos fundamentales"4 o "derechos básicos", 5 no es fácil encapsular en una

\footnotetext{
2 De J. G. FICHTE, Grundlage Des Naturrechts Nach Prinzipien Der Wissenschaftslehre, Nabu Press, 2011; a J. FinNis, Natural Law and Natural Rights, Second Edition, Oxford University Press, Oxford, New York, 2011.

3 De Asamblea General de las NACiONES Unidas, "Declaración Universal de Derechos Humanos" París, 10 de diciembre de 1948, Resolución 217 A (III); a R. CRUFT; S. M. LIAO; M. Renzo (EDS.), Philosophical Foundations of Human Rights, Oxford University Press, Oxford, New York, 2015.

4 De Union EuRopeA, Carta de los Derechos Fundamentales de la UE, 201014 de diciembre de 2007 (reformada), en vigor 1 de diciembre de 2009 (junto con el Tratado de Lisboa); a G. Peces-Barba Martínez; E. Fernández García; R. De Asís Roig; C. R. Fernández Liesa; F. J. Ansuátegui Roig (eds.), Historia de los derechos fundamentales, Dykinson, Madrid, 2003.
} 
expresión la noción básica que alberga el cerebro del Homo sapiens: ${ }^{6}$ la necesidad de tener algunos derechos de los que no se puede privar a un ser humano.

Una revisión de la literatura jurídica desde la Edad Moderna, remontándonos a Bartolomé de las Casas $^{7}$ y llegando a los filósofos más innovadores de nuestros días, ${ }^{8}$ muestra una búsqueda continua de los fundamentos de los derechos de los que depende la esencia de la vida humana. El objetivo de este artículo es doble. Por un lado, explorar qué instrumentos nos puede ofrecer el análisis económico en esta búsqueda. ${ }^{9}$ Por otro, ofrecer un contraargumento a la crítica "relativista". ${ }^{10}$

A los efectos de este ensayo, entendemos la "crítica relativista" en su vertiente más simplificada y tradicional, ${ }^{11}$ conforme a la cual es imposible justificar la existencia de derechos "universales", porque el derecho se

\footnotetext{
${ }^{5}$ Utilizando la terminología de H. SHUE, Basic Rights: Subsistence, Affluence, and U.S. Foreign Policy, Princeton University Press, Princeton, 1996; recogida también por C. R. BeITZ; R. E. Goodin, Global Basic Rights, Oxford University Press, New York, 2009.

${ }^{6}$ Esta afirmación puede parecer excesiva dado su carácter generalizador. Sin embargo, se deriva de manera lógica del desarrollo de la empatía en humanos como un trazo evolutivo y biológico, de la manera sugerida por Hoffman desde los años ochenta y hoy relativamente aceptada en mayor o menor grado. Si somos seres empáticos por naturaleza, y los desajustes de dicha empatía se consideran enfermedades (por ejemplo en psicopatías), parece coherente asumir que nuestro cerebro esta configurado para sentir la necesidad de que todos recibamos un cierto nivel de "trato justo". Ver en este sentido M. L. Hoffman, Empathy and Moral Development: Implications for Caring and Justice, Cambridge University Press, 2001, pp. 4, 8. Una visión relativamente equilibrada sobre lo probado y lo probable sobre el determinismo jurídico a nivel neuronal puede verse en la crítica de Scull a S. ZeKI; O. GoOdenOUGH (EDS.), Law and the Brain, Oxford University Press, Oxford, New York, 2006 publicada en Brain, una de las principales revistas de neurociencia. Ver A. SCULL, "Mind, brain, law and culture", Brain, 130, 2,
} 2007.

${ }^{7}$ Ver por ejemplo M. BEUCHOT, Los fundamentos de los derechos humanos en Bartolomé de las Casas, Anthropos Editorial, 1994.

${ }^{8}$ O'Manique, por ejemplo, justifican la existencia de los derechos esenciales en la biología evolutiva, lamentando que autores clásicos como Rawls o Kant se centrasen en justificaciones teóricas. Lamentablemente, idéntica crítica se puede dirigir a este artículo. Ver J. O'MANIQUE, "Universal and Inalienable Rights: A Search for Foundations", Human Rights Quarterly, 12, 4, 1990; J. O'MANIQUE, The Origins of Justice: The Evolution of Morality, Human Rights, and Law, University of Pennsylvania Press, 2003.

9 Trabajos anteriores con similares postulados como el de Veseth estaban enfocados en exceso a la supuesta contradicción entre derechos de base económica y otros derechos inalienables M. VESETH, "The Economics of Property Rights and Human Rights", American Journal of Economics and Sociology, 41, 2, 1982.

${ }^{10}$ Por todos, véase la declaración de la Asociación Americana de Antropología emitida durante los trabajos preparatorios de la Declaración Universal de los Derechos Humanos: "Standards and values are relative to the culture from which they derive so that any attempt to formulate postulates that grow out of the beliefs or moral codes of one culture must to that extent detract from the applicability of any Declaration of Human Rights to mankind as a whole." A. A. A. The EXECUTIVE BOARD, "Statement on Human Rights", American Anthropologist, 49, 4, 1947.

11 Nos alejamos así de aquellos autores que han tratar de conciliar las objeciones relativistas "moderadas" con la existencia de derechos universales a través de la modulación de su ejercicio, sin negar la existencia de derechos estrictamente universales. 
crea en el marco de una sociedad dada a partir de ciertos valores particulares de esa sociedad. ${ }^{12}$

La conclusión principal de este artículo es que a cualquier ser humano racional desde el punto de vista económico le gustaría vivir en una sociedad que garantice algunos derechos, independientemente de todo lo demás. Este razonamiento supera cualquier posible crítica libertaria, ya que es el resultado de una elección individual egoísta, no restringida por ninguna imposición externa.

El artículo se divide en tres partes. En la primera se realiza una justificación de la necesidad de buscar una fundamentación diferente a las ya existentes. En esta sección se describen también las limitaciones de esta propuesta en términos teóricos y de utilidad práctica. La segunda parte es un recorrido a través de siete pasos (o asunciones) para llegar a la conclusión de la existencia de un fundamento económico racional de ciertos derechos inalienables. En la parte final se presentan unas observaciones a modo de conclusión que tratan de anticipar críticas a esta propuesta.

\section{LA BÚSQUEDA DE UNA NUEVA FUNDAMENTACIÓN PARA LOS DERECHOS INALIENABLES: ¿POR QUÉ Y PARA QUÉ?}

La principal contribución de este artículo, es decir la existencia de un fundamento económico para ciertos derechos inalienables, tiene una clara vertiente política desde una perspectiva internacionalista. Dada la dinámica contemporánea del sistema global, la crítica relativista ha pasado de ser una crítica filosófica, a convertirse en una poderosa herramienta política. Desde esta perspectiva, los derechos inalienables serían contextuales.

Sirva aquí de ejemplo, la referencia a ciertos "valores asiáticos" como "moderadores" de estos derechos inalienables. ${ }^{13}$ Esta idea ha sido objeto de recurrente debate en tiempos recientes, a pesar lo discutido del concepto en sí mismo. ${ }^{14}$

Probablemente el mejor representante de la crítica a la idea de los valores asiáticos la haya presentado el (asiático) premio Nobel de economía Amartya Sen en una conferencia ya clásica en 1997 donde desafió la idea en sí misma de que puedan existir unos valores "regionales" en un continente tan diverso; o que, de existir, estos pudiesen ejercer un impacto sustancial sobre los derechos inalienables. ${ }^{15}$

Sin embargo, la negación de la mayor -o de la base fáctica de la crítica- es insuficiente. Puede servir en un debate "occidentalizado" pero resulta a todas luces inoperativa cuando el debate se establece en

12 En cierta manera aproximaciones a la naturaleza del derecho como la teoría tridimensional de Reale descansan en esta idea de relación entre norma, hecho y valor. Véase M. REALE, Teoria tridimensional do direito, Saraiva, São Paulo, 1994.

13 D. Kingsbury, "Universalism and Exceptionalism in "Asia"", en Human Rights in Asia, Springer, 2008.

${ }^{14}$ L. JENCO, "Revisiting Asian Values", Journal of the History of Ideas, 74, 2, 2013.

${ }^{15}$ A. K. Sen, "Democracy as a universal value", Journal of democracy, 10, 3, 1999. 
términos culturales en los que la mera negación del relativismo puede ser considerada inadecuada desde un punto de vista político o filosófico. ${ }^{16}$

De análoga manera, aquellos enfoques alternativos que tratan de establecer fundamentos universales son igualmente problemáticos. Más allá de la idea de derechos otorgados por un dios o por la naturaleza, cualquier otro fundamento o argumento existente puede verse como culturalmente limitado. Siguiendo la tipología de Shestack, podemos analizar uno a uno los principales fundamentos utilizados a lo largo de los últimos doscientos años y ver su relación con los elementos culturales subyacentes. ${ }^{17}$

Así, la autoridad del Estado depende de una construcción cultural: el Estado mismo. La idea de la existencia de un carácter naturalmente social en el ser humano, fundamento de las construcciones marxistas, son un desarrollo teórico en un marco preciso, como la industrialización, en un contexto cultural determinado, como lo es la problemática de clases en la Europa del XIX. El utilitarismo clásico o la maximización de la felicidad agregada requiere una autoridad dominante que pueda evaluar las concesiones individuales en aras del bienestar de la colectividad, es decir, otro tipo de construcción social. Los enfoques basados en valores, como "justicia", "reacción a la injusticia" o "igualdad" pueden significar cosas diferentes en diferentes idiomas o entornos. "Dignidad" (humana), tal vez el valor más recurrido como fundamento, ${ }^{18}$ no solo presenta defectos de traducción a lenguas donde dicho término no se deriva del latín, sino que descansa también en un aura de "supervalor" atribuido a la dignidad que la acerca peligrosamente al enfoque utilizado por la doctrina del derecho natural. ${ }^{19}$

Si no se pudiese encontrar un fundamento realmente universal, una opción alternativa sería aceptar la crítica, e incorporarla en la configuración de los derechos humanos, al menos de manera parcial. Podríamos entender así que "la universalidad antropológica y ontológica es empírica, filosófica o políticamente indefendible" a cambio de dotar de un espacio a los derechos humanos pero bajo el corsé de un "desarrollo nacional, regional y cultural particular y de otras formas de diversidad y relativismo". ${ }^{20}$ Sin embargo ello nos expondría a un diabólico debate sobre cuánta relatividad es aceptable antes de que los derechos inalienables dejen de ser universales.

\footnotetext{
${ }^{16}$ Véase por ejemplo la presentación de esta cuestión al inicio del libro de A. D. RENTELN, International Human Rights: Universalism Versus Relativism, Quid Pro Books, 2013, p. 1.

17 J. J. SHESTACK, "The Philosophic Foundations of Human Rights", Human Rights Quarterly, 20, 2, 1998; y J. J. SHESTACK, "The philosophic foundations of human rights", en Human Rights: Concept and Standards, Routledge, Abingdon \& New York, 2016,

${ }^{18}$ Entre otros, en esta propia revista por M. E. DE LOS RIOS URIARTE, "Las dimensiones trascendentes de la dignidad humana como fundamento para la formulación de los derechos humanos.", Dereito, 23, 1, 2013.

19 J. J. SHESTACK, "The philosophic foundations of human rights", cit., pp. 53-54.

20 J. DonNelly, "The Relative Universality of Human Rights", Human Rights Quarterly, 29, 2, 2007, p. 281.
} 
Por ello necesitamos encontrar fundamentos comunes en nuestras visiones filosóficas. La economía neoclásica y sus principios podrían ser uno de ellos, ya que muchos de los defensores de esos valores asiáticos no tienen problemas en admitir la idea de que un ser humano puede ser económicamente racional.

Este artículo propone ese acercamiento en términos simples y estructurado en pequeños pasos fácilmente comprensibles para lectores ajenos a la economía y a la formalización matemática, muchas veces excesiva en artículos desarrollados en la marco del análisis económico del derecho.

Debemos ser igualmente claros sobre qué no es este trabajo. No es un artículo de Filosofía, con mayúscula. No es tampoco un artículo de análisis económico formal. Los principales destinatarios de este trabajo son profesionales en el campo de las relaciones internacionales y el derecho internacional. Está escrito con el objetivo de ayudar a activistas y abogados de derechos humanos, diplomáticos, funcionarios de organizaciones internacionales y trabajadores del sector de desarrollo que se enfrentan a un nivel teórico con los obstáculos creados en su labor diaria por el relativismo cultural en materia de derechos humanos.

En el terreno sustantivo, es necesario notar aquí una importante advertencia: el enfoque presentado aquí es un enfoque y solo uno, entre muchos otros. Su objetivo no es promover el "imperialismo económico" u ocupar el espacio teórico en materia de derechos inalienables desplazando a otras aproximaciones hacia ellos que puedan responder mejor a la naturaleza humana que el razonamiento económico. Su intención es tan solo acercar algunas herramientas que han sido significativamente ignoradas, al lector interesado en este debate.

Finalmente, debe señalarse que las conclusiones aquí presentadas tienen un alcance limitado, dejando fuera de su ámbito de aplicación a derechos tradicionalmente considerados esenciales en el marco de los derechos inalienables. Se concluye también aquí que la distinción tradicional entre derechos civiles y políticos, por un lado, y derechos económicos, sociales y culturales, por otro, es inadecuada desde un punto de vista lógico-formal. Esta idea no es en absoluto novedosa, pero su justificación en este artículo difiere significativamente de otros argumentos antes presentados. ${ }^{21}$ En la práctica, ello significa que los derechos aquí identificados como inalienables pueden recaer en cualquiera

${ }^{21}$ Esta distinción (o de primera y segunda generación) es considerada obsoleta por muchos, véase por ejemplo H. V. CondÉ, A Handbook of International Human Rights Terminology, University of Nebraska Press, Lincoln, 2004, p. 236. Sin embargo es frecuente encontrar disquisiciones doctrinales sobre sus diferencias en ciertos contextos académicos, véase por ejemplo B. OREND, Human Rights: Concept and Context, Broadview Press, 2002, p. 110. En cualquier caso la terminología y el enfoque diferenciado son comúnmente utilizados por profesionales de los derechos humanos, probablemente debido a la influencia de su base jurídica: el Pacto Internacional de Derechos Civiles y Políticos (Nueva York, 16 de diciembre de 1966), vigente (excepto el artículo 41) desde el 23 de marzo de 1976; y el Pacto Internacional de Derechos Económicos, Sociales y Culturales (Nueva York, 16 de diciembre de 1966), en vigor desde el 3 de enero de 1976. 
de las dos categorías anteriores, mientras que algunos de los derechos tradicionalmente enumerados en ambos no pueden considerarse derechos inalienables desde una perspectiva económica.

\section{SIETE PASOS PARA SENTAR LAS BASES ECONÓMICAS DE LOS DERECHOS INALIENABLES}

\subsection{El ser humano es un Homo economicus}

A pesar de la clásica "objeción reduccionista", ${ }^{22}$ la idea de que el ser humano es un Homo economicus es una suposición convencional en la economía neoclásica. ${ }^{23}$ Podría ser - de hecho, es- mucho más que un Homo economicus, pero ello no afecta al principio de que todo hombre o mujer debe enfrentarse a un mundo de recursos limitados en el que debe sobrevivir. Como Posner, uno de los principales autores en la aplicación del razonamiento económico a otras áreas del conocimiento, expresó de manera elocuente: "elegir los mejores medios para el fin determinado por aquel que elige"24 parece ser un principio razonable de conducta general.

Sin embargo, una gran cantidad de estudios sobre comportamiento económico han demostrado que los humanos no actuamos de manera racional en muchas ocasiones. ${ }^{25} \mathrm{~A}$ pesar de ello, podemos estar de acuerdo en que la racionalidad (económica) es una guía razonablemente adecuada para determinar (¿predecir tal vez?) comportamientos esperados. ${ }^{26}$ El cerebro humano ha sido esculpido a través de su evolución para enfrentarse a este hecho. De alguna manera, reside en nosotros un gestor que trata de maximizar nuestro bienestar personal aplicando el concepto de utilidad marginal.

22 Debido a las acusaciones de imperialismo económico dirigidas a la escuela de la Elección Pública, merece la pena notar aquí las observaciones sobre el Homo economicus de dos de sus más importantes figuras: "Our plea is the more modest one that calls upon our fellow economists to recognize that the Homo econonomicus has its own limits as a useful abstraction" J. M. BUCHANAN; R. D. TOLLISON, The Theory of public choice--II, University of Michigan Press, 1984, p. 384. Particularmente interesante es la crítica feminista. Un ejemplo de la misma pude encontrarse en M. Fineman; T. DougherTY, Feminism confronts homo economicus: gender, law, and society, Cornell University Press, 2005. Para una revision de las críticas y un análisis de su origen debe consultarse J. PERSKY, "The ethology of homo economicus", Journal of Economic Perspectives, 9, 2, 1995, pp. 221-224.

${ }_{23}$ Véase por ejemplo el discurso de Stigler en las Lecciones Tanner de 1980, notablemente sus observaciones finales. G. STIGLER, "Economics or ethics?", 1980, Harvard University, fecha de consulta en https://tannerlectures.utah.edu/_documents/ato-z/s/stigler81.pdf.

24 "Choosing the best means to the chooser's end" R. A. POSNER, "Rational Choice, Behavioral Economics, and the Law", Stanford Law Review, vol. 50, 5, 1998, p. 1551.

${ }^{25}$ Thaler describe esta situación de una manera humorística en el resumen de un artículo ya clásico: "My specific predictions are that Homo Economicus will start to lose IQ, will become a slower learner, will start interacting with other species, and that economists will start to study human cognition, human emotion, and will distinguish more clearly between normative and descriptive theories" R. H. THALER, "From Homo Economicus to Homo Sapiens", Journal of Economic Perspectives, vol. 14, 1, 2000.

26 Por todos, G. S. BeCKer, "Altruism, Egoism, and Genetic Fitness: Economics and Sociobiology", Journal of Economic Literature, vol. 14, 3, 1976. 
Sin embargo, nadie debería entender "nuestro" como "simplemente mío". Otro rasgo de la naturaleza humana es obtener algún tipo de "beneficio" al hacer que algunas de las personas que nos rodean "estén mejor". En el contexto de la premisa descrita en el párrafo anterior, ello significa que "nuestra utilidad o bienestar personal" podría ir más allá de las consecuencias esperadas para nosotros mismos e incluir espontáneamente el efecto de nuestras acciones en los demás.

Algunos autores como Becker han solucionado esta cuestión incluyendo el comportamiento altruista al definir la función de utilidad de los individuos, ${ }^{27}$ pero la idea se remonta, al menos, a John Stuart Mill. ${ }^{28}$

En cualquier caso, que nuestra decisión tome o no en cuenta las consecuencias para el bienestar de los demás, más allá del propio, no afecta a la lógica del razonamiento económico: los recursos seguirán siendo limitados y el ser humano deberá emplear ciertos recursos, al menos en una cantidad mínima que pueda asegurar su supervivencia y la de su especie. Por otro lado, la elección no dejar de ser individual por incorporar en nuestra decisión cómo nuestras acciones afectarán a otros. Es más, puede incluso que un comportamiento que tenga en cuenta las repercusiones sobre los demás tenga una base puramente egoísta. Un ejemplo de ello sería una conducta decidida en base a la previsión de una situación inversa en la que nos pongamos en lugar del otro y que nuestra actitud sea una especie de expectativa de quid pro quo.

\subsection{El ser humano nunca se enfrenta a una decisión sobre qué derechos deberían ser sus derechos inalienables}

Cuando nacemos, o somos concebidos -en función de nuestras creencias- no se le da a uno la opción de elegir entre un conjunto de derechos que nos gustaría tener. La sociedad en la que nacemos ha tomado colectivamente esa decisión por nosotros. En principio, este paso no parece exigir mayor desarrollo para justificarlo adecuadamente, si bien requiere una precisión.

No se quiere decir aquí que realmente haya existido en cualquier sociedad dada un procedimiento colectivo de elección. A los efectos de este razonamiento, se hace esta afirmación de una manera mucho más limitada. Entre las combinaciones (casi) infinitas de derechos inalienables que podrían existir, la sociedad en la que nacemos cuenta - "ha optado" en sentido laxo- por un conjunto dado de ellas. Este conjunto puede cambiar a medida que crecemos en esa sociedad $y$, obviamente, en algún momento nuestras acciones pueden influir en esa elección. Sin embargo $-y$ he aquí lo relevante a los efectos de nuestra exposición- cuando nacemos no hemos tenido la oportunidad de participar en dicha elección.

${ }^{27}$ G. S. BECKER, "Altruism in the Family and Selfishness in the Market Place", Economica, vol. 48, 189, 1981.

${ }^{28}$ Brown realiza un análisis de las implicaciones filosóficas de esta cuestión en D. G. Brown, "What Is Mill's Principle of Utility?", Canadian Journal of Philosophy, vol. 3, 1, 1973, p. 8. 


\subsection{El velo de la ignorancia rawlsiano es una herramienta útil para sobrepasar esa imposibilidad intrínseca}

Por bien dirigidas o acertadas que hayan sido las críticas liberales a las construcciones rawlsianas, ${ }^{29}$ no es menos cierto que el velo de ignorancia de Rawls nos puede proporcionar un mecanismo muy útil para recrear a nivel teórico la situación de preexistencia.

Si entendemos "justicia como equidad", ${ }^{30}$ como hace Rawls, parece coherente pensar que los principios primigenios de justicia deberían ser adoptados como un acuerdo original entre personas racionales ${ }^{31}$ en posición de igualdad. Estas personas establecerían un acuerdo entre ellos que regularía los términos básicos de su asociación.

Consecuentemente, y a efectos de coherencia, el diseño de Rawls demanda un cierto grado de decisión colectiva. Así, para su funcionamiento debe cumplirse el siguiente requisito: tenidas en cuenta las circunstancias de las partes, y sus conocimientos, creencias e intereses, la mejor manera para que cada persona asegure sus fines, dadas las alternativas disponibles, es un acuerdo sobre estos principios. ${ }^{32}$

Para los efectos de este artículo, el velo de Rawls es, no obstante, insuficiente. En primer lugar, nuestra construcción teórica requiere una completa aleatoriedad. En este escenario nadie debe saber en qué posición social se encontrará, ni en qué etapa de su vida, ni siquiera en qué sociedad. Esta última es tal vez la más evidente diferencia con la construcción del jurista estadounidense que parece restringida a un sistema contemporáneo y occidentalizado. Podríamos incluso imaginar una sociedad asocial (si se permite el oxímoron) donde puede existir tan solo un contacto fortuito con otros seres humanos. ${ }^{33}$ La ceguera, al optar por el tipo de derechos que un individuo dado desearía "en abstracto", sería así completa. ${ }^{34}$

\footnotetext{
${ }^{29}$ Ver, por ejemplo R. Nozick, Anarchy, State, and Utopia, Basic Books, 1977. Una interesante discusión sobre el impacto que el debate entre estos autores ha tenido para la posteridad puede encontrarse en A. CHANDER; A. CHANDER, "Is Nozick Kicking Rawls's Ass - Intellectual Property and Social Justice Symposium: Intellectual Property and Social Justice: Foreword", U.C. Davis Law Review, vol. 40, 2006.

${ }^{30} \mathrm{~J}$. RAWLS, "Justice as fairness", The philosophical review, vol. 67, 2, 1958.

${ }^{31}$ El requisito de racionalidad plantea una dificultad en aquellos casos en los que las capacidades intelectuales de una persona se ven afectadas de manera que limiten su capacidad de realizar el proceso descrito en los pasos siguientes. Los casos más paradigmáticos serían el de una persona con una discapacidad psíquica pronunciada o el de una persona que sufriese un trastorno mental grave. Se discute en más detalle estas situaciones en las observaciones finales.

32 J. RAwLS, A Theory of Justice, Oxford University Press, 1999, pp. 102-103.

${ }^{33}$ Los seres humanos son, por naturaleza, seres sociales. Por lo tanto, pensar en un Homo economicus completamente separado de (cualquier) sociedad no solo no es realista, sino que rozaría el absurdo. Sin embargo, esta es la única manera posible de garantizar que el velo de ignorancia sea completo y que las decisiones del Homo economicus no estén influenciadas por sus expectativas aprendidas.

34 Esta configuración del velo de ignorancia nos acerca al "velo de incertidumbre" desarrollado por los economistas constitucionales. Sin embargo, la suposición de los economistas constitucionales es la existencia de una negociación para crear una sociedad en la cual todos sus miembros deben estar de acuerdo (condición de unanimidad) para
} 
La segunda diferencia, tal vez menos obvia, es que nuestro velo no da lugar a un proceso de construcción colectiva en el que todos deben salir ganando cooperando. Muy al contrario, nuestro velo se restringe a un cálculo personal y egoísta, como veremos más abajo.

No es difícil observar que este escenario adolece de una falta total de verosimilitud, en tanto que nuestra reflexión es puramente intelectual y puede recordar en exceso a muchos otros desarrollos de las construcciones rawlsianas.

En cualquier caso, para aquellos que no disfrutan de ejercicios teóricos, se podría sugerir que imaginen la invención de un compuesto revolucionario que permite la vida casi eterna, pero que no funciona para todos. En esta situación, el muy largo plazo, la incertidumbre necesariamente aparejada a una esperanza de vida muy larga, haría más "tangible" la necesidad de arbitrar un conjunto de derechos inalienables, antes de decidirse a tomar (o no) ese compuesto.

Así configurado, nuestro punto de partida es un escenario en el que cada ser humano tiene la capacidad de decidir bajo qué conjunto de reglas le gustaría vivir, ignorante de su posición dentro de la sociedad o, incluso, en qué tipo de sociedad vivirá.

\subsection{El Homo economicus desea suavizar el consumo}

Cualquier individuo prefiere trasferir el consumo de períodos cuando este es alto $-y$, por lo tanto, con una utilidad marginal baja- a períodos cuando es bajo $-y$, opuestamente, con utilidad marginal alta.

La explicación de este principio generalmente aceptado en la economía neoclásica es bastante simple, pero tal vez sea más útil ilustrarlo con un ejemplo. Supongamos que tengo que decidir cómo distribuir a lo largo de mi vida $100 \mathrm{~m} 3$ de agua. La opción extrema sería tener $100 \mathrm{~m} 3$ de agua un día y ninguno en otro momento de mi vida. Lo opuesto a esta concentración de utilidad en un solo día, sería distribuirlo a la largo del tiempo, por ejemplo, a razón de 3 litros por día. Ello es así, incluso si sucede que no vivo lo suficiente como para disfrutar de mis $100 \mathrm{~m} 3 .^{35}$

Incluso si los $100 \mathrm{~m} 3$ me permitieran llenar la piscina (que podría tener la suerte de tener también) y disfrutar de un gran primer día de vida, mi aversión al riesgo generada por la posibilidad de no tener un bien esencial durante el resto de mi vida, haría que mi mente racionalmente económica considerase optar por los 3 litros diarios como una mejor

establecerla. No se trata aquí de analizar este paso, concentrando su atención en cómo cualquier Homo economicus debe decidir autónomamente cuáles son sus derechos inalienables. En este escenario que no se lleva a cabo ninguna negociación. Para una crítica bien fundamentada que, no sería fácilmente aplicable en nuestra situación hipotética, sino para el "marco general de incertidumbre", véase C. MüLLER, "The Veil of Uncertainty Unveiled", Constitutional Political Economy, vol. 9, 1, 1998.

${ }^{35}$ Para ahorrar la molestia de hacer los cálculos, los $100 \mathrm{~m} 3$ originales me durarían más de 33.333 días, que son más de 91 años. 
opción. Ello incluso a pesar de tener que ver mi piscina vacía todos los días. Esta idea puede formalizarse de manera muy básica

Imaginemos que mi utilidad fue definida (arbitrariamente) por una función como $U=\sqrt{ } \mathrm{C}$, siendo " $U$ " mi utilidad y "C" mi consumo de agua por día. Si sé que voy a vivir 91 años y consumo mis 100.000 litros (100 $\mathrm{m} 3$ ) en un día, mi utilidad esperada sería $\sqrt{ } 100,000+\sqrt{ } 0+\sqrt{ } 0+\ldots+$ $\sqrt{ } 0$, es decir, $316^{\prime} 23$. Si opto por el enfoque de suavizar mi consumo, mi utilidad sería $\sqrt{3}+\sqrt{3}+\sqrt{ } 3+\ldots$ o simplemente para ahorrarnos algo de tiempo y papel $33.333 \times \sqrt{ } 3$, es decir, 57.334'45. En conclusión, suavizar el consumo nos proporciona, a largo plazo, una mayor utilidad.

En cualquier caso, esta formalización debe acomodar también ciertas situaciones hipotéticas:

(i) ¿Qué sucede si muero antes de llegar al punto en que mi utilidad llegue a $316^{\prime} 233^{36}$ En principio, ello no debería afectar a la decisión que tomo el primer día $\left(t_{1}\right)$ o, para ser más precisos, antes del primer día $\left(t_{0}\right)$ de suavizar el consumo. Aun siendo plenamente consciente del riesgo de morir antes del día $183\left(\mathrm{t}_{183}\right)$, preferiría los 3 litros al día. Ello es así porque la elección es adoptada en base a la utilidad esperada. No se trata de la utilidad real que se obtiene ex post, sino de lo que se espera obtener de una decisión ex ante.

(ii) ¿No deberíamos tener en cuenta las probabilidades de morir? Efectivamente. En una situación de información perfecta, mi decisión racional se debe ver afectada por las probabilidades que sé que tengo de morir. Por una cuestión de simplificación, no se incorporó el efecto de la probabilidad en la formulación anterior, asumiendo por ello que se iban a vivir los 91 años completos. De hecho, la utilidad esperada (UE) se formalizaría de manera adecuada como

$$
U E=(1-p) \times U+p \times U
$$

$\mathrm{UE}=$ Consumo sin eventos adversos + consumo con eventos adversos

No obstante, asumiendo que las probabilidades de morir son las mismas cada día (distribución proporcional), la inclusión del factor probabilidad no alteraría el resultado: Me gustaría "asegurarme completamente" contra el riesgo de acabar el agua antes de acabar mi vida.

Sin embargo, si supiera que las probabilidades de morir antes del día 183 son realmente altas, tal vez decida optar por el día en la piscina, pero, una vez más, debemos tener en cuenta que no sabríamos lo que vendría primero: la muerte por deshidratación o el "día de piscina" salvo que optásemos por el disfrute de los $100 \mathrm{~m} 3$ el primer día.

(iii) ¿Por qué la función se define como $\mathrm{U}=\sqrt{ } \mathrm{C}$ y no como cualquier otra que pudiese llevarnos a una conclusión diferente? Como señalamos con anterioridad, determinamos esta función de utilidad de una manera completamente arbitraria, siendo la única restricción que la utilidad marginal debe ser decreciente. El principio de "utilidad marginal decreciente" implica que cualquier incremento absoluto en el consumo

\footnotetext{
${ }^{36}$ Nuevamente, para ahorrarle algo de tiempo al lector, eso sucedería el día $183 \mathrm{si}$ consumo mis 3 litros por día.
} 
tiene que conducir a un incremento menor en proporción de la utilidad. Por continuar con el ejemplo del agua, cada litro de agua extra al día debe aportarnos menor utilidad que el anterior. Este principio es un axioma básico de la economía neoclásica, ${ }^{37}$ doctrina de referencia en el grueso de la dicha disciplina y en ocasiones llamada, precisamente, "escuela marginalista". ${ }^{38}$ La puesta en cuestión de esta asunción implica un desafío a uno de los fundamentos de la economía moderna. Una vez que se respeta la regla de la utilidad marginal decreciente, cualquier función que se pueda imaginar conduciría a la misma conclusión.

\subsection{En la situación de preexistencia, la elección de un conjunto dado de reglas fundamentales de la comunidad puede verse como un mercado}

Si vemos la elección sobre qué restricciones nos queremos imponer en nuestro comportamiento futuro como un coste, podemos entender que la moneda de pago es a qué parte de nuestra libertad natural estamos dispuestos a renunciar.

Para poder utilizar adecuadamente esta premisa debemos añadir aquí otra suposición. Al decidir qué nivel de restricciones nos queremos imponer, no tenemos defectos en nuestra información, ${ }^{39}$ es decir, entendemos adecuadamente las restricciones a las que nos sometemos. De hecho, en este escenario la única cosa que no sabemos es en qué situación nos vamos a encontrar, pero conocemos nuestras necesidades mínimas desde el nacimiento hasta la muerte. Esto, sin duda, no es plausible, pero dado que ya hemos colocado al Homo economicus detrás del velo de la ignorancia, un grado adicional de irrealidad sería poco perjudicial y nos ayudaría a superar una de las críticas más sólidas a la existencia misma del Homo economicus, como es la ausencia de información adecuada en la toma de sus decisiones.

En este punto, nos encontramos también otro obstáculo: ¿Cómo podemos diferenciar entre mi estándar subjetivo de aversión al riesgo y el estándar mínimo que comparte cada Homo economicus? O, reformulando la pregunta, ¿no podrían dos personas racionales y bien informadas llegar

\footnotetext{
37 Sobre los principios en los que descansa la economía neoclásica y sus limitaciones en general, se puede consultar en castellano G. MAYA MUÑOz, "La teoría neoclásica: reflexiones.", Ensayos de Economía, vol. 4, 7, 1993. En inglés, es clásico el artículo de Nagel sobre las ideas de Friedman. E. NAGEL, "Assumptions in Economic Theory", The American Economic Review, vol. 53, 2, 1963.

${ }^{38} \mathrm{Si}$ bien algunos distinguen entre ambas escuelas (ver, p.ej. T. Aspromourgos, "On the origins of the term «neoclassical»", Cambridge Journal of Economics, vol. 10, 3, 1986), el consenso desde el punto de vista del uso habitual es la existencia de una única escuela neoclásica-marginalista con diferentes tendencias. En cualquier caso, el término "neoclásico" es en sí problemático y ha sido declarado "oficialmente muerto" por el Presidente de la Sociedad de Historia de la Economía. D. ColAnder, "The Death of Neoclassical Economics", Journal of the History of Economic Thought, vol. 22, 2, 2000.

39 Esta es otra de las restricciones que la economía neoclásica impone al Homo economicus. Para una lista detallada de las mismas, ver de nuevo G. STIGLER, "Economics or ethics?", cit. nota 23.
} 
a conclusiones diferentes sobre qué derechos debería garantizar la sociedad?

Es razonable pensar que, estando detrás del velo de ignorancia, cualquier persona aceptaría ciertas reglas básicas, como, por ejemplo, no ser privado de su vida sin razón. Sin embargo, parece igualmente razonable asumir que dos personas puedan optar por un estándar de atención sanitaria más o menos comprensivo ¿No son ambas opciones perfectamente legítimas y lógicas desde un punto de vista económico?

Este argumento es, de hecho, una de las cuestiones más debatidas en las teorías de Rawls: cómo acomodar diferentes niveles de aversión al riesgo o, de manera más explícita ¿cómo lidiamos con aquellos casos en los que exista la posibilidad de grandes ganancias a cambio de haber aceptado un pequeño riesgo de pérdidas? ${ }^{40}$

\subsection{Todo individuo estaría de acuerdo con estar completamente asegurado contra algunos de los riesgos}

La regla de oro para revelar estas preferencias individuales es relativamente simple pero igualmente efectiva: ¿en qué medida un individuo económicamente racional está dispuesto a asumir algún riesgo? Cuando la respuesta de todos los miembros sea "ninguna" -o más precisamente "cuando la respuesta del Homo economicus ficticio que todos compartimos sea 'ninguna'"- la regla que protege a un individuo de ese riesgo específico tiene necesariamente que ser una norma fundamental de la sociedad.

Esto es diferente del mercado de seguros imaginado por Dworkin. ${ }^{41} \mathrm{~A}$ grandes rasgos, el escenario ideado por este filósofo del derecho es un sistema de redistribución, donde cada persona podría comprar un seguro contra la posibilidad de nacer (levantar el velo) con algún tipo de limitación que afectase a su capacidad de generar rentas en una sociedad dada. Si ello sucediese, esta persona recibiría un pago de la sociedad. El escenario de Dworkin funcionaría también a la inversa pagando a la sociedad si se naciese con ciertos talentos altamente comercializables.

El razonamiento que aquí nos ocupa sirve en cambio para identificar aquellos casos en los cuales ningún nivel de pago sería suficiente. En otras palabras, nos enfrentamos a situaciones en las cuales, si sucediese ese evento, no habría compensación posible. El daño sería tal que preferiría no ser parte de esa sociedad en absoluto. Partiendo de este razonamiento podríamos determinar cuáles son esas situaciones y las normas fundamentales que los protegen.

La manera más intuitiva de formalizar este paso es viéndolo como un mínimo común denominador de las preferencias individualizadas. Si cada Homo economicus establece sus requisitos para formar parte de la

\footnotetext{
${ }^{40}$ J. RAWLS, "Reply to Alexander and Musgrave", The Quarterly Journal of Economics, vol. $88,4,1974$.

${ }^{41}$ R. DWORKIN, "What is Equality? Part 2: Equality of Resources", Philosophy \& Public Affairs, vol. 10, 4, 1981.
} 
sociedad de manera individual, tendríamos un mínimo común en el punto más bajo de derechos establecidos.

\subsection{Cuando cada individuo desea asegurarse de manera completa e incondicional, la norma que establece ese seguro protege un derecho inalienable}

En el paso descrito en el apartado anterior hemos establecido un mecanismo para identificar qué riesgos son insoportables para el Homo economicus. Consecuentemente, la norma que protege a todos los individuos de un riesgo específico tiene que ser necesariamente una norma fundamental de cualquier sociedad. Agregamos ahora que el derecho subyacente protegido por dicha norma es inalienable. Existe, por lo tanto, una equivalencia entre (i) un "seguro incondicional en cualquier estado del mundo" y (ii) "derechos inalienables".

Para aquellos con una mente más gráfica, es posible representar la relación de intercambio entre $(x)$ el grado de restricción de libertad original dispuesto a aceptar; e (y) el nivel de riesgo que estaría dispuesto a aceptar de que el evento adverso ocurra. Cuando dicha relación de intercambio se plasma en una línea recta paralela al nivel en el que el riesgo que estamos dispuestos a aceptar de que ocurra el evento adverso es ninguno, podemos concluir que nos encontramos ante un riesgo inasumible. Dicho riesgo debe ser evitado a través de una norma fundamental y, por lo tanto, estaríamos protegiendo con esa norma un derecho inalienable.

Todo ello, siempre y cuando la restricción de la libertad original que pagamos como coste no afecte a otra área en la que deseemos estar también completamente asegurados.

Se deriva de ello que la curva de utilidad personal del cualquier Homo economicus sería una línea recta que representa un "seguro incondicional". Asumiendo que hay un "estado del mundo" en el cual el evento adverso tiene algunas probabilidades de surgir, cualquier individuo racional maximizaría su utilidad estando completamente asegurado. Ello es así porque el costo de que ese evento ocurra sería insoportable.

\section{OBSERVACIONES A MODO DE CONCLUSIÓN}

A partir de los siete pasos anteriores hemos desarrollado un mecanismo para determinar qué es un derecho inalienable en base a una fundamentación económica. Nótese, que se ha desarrollado una definición puramente formal de lo que es un derecho inalienable; es decir, la única manera de asegurar que algo es un derecho inalienable es determinando primero la existencia de una norma que protege al Homo economicus contra un riesgo insoportable.

En este sentido los "derechos inalienables" que se describen aquí no se determinan sobre la base de su contenido sustantivo o porque protegen bienes superiores (incluso si estos lo son). Son inalienables, en su sentido puramente etimológico, ya que no pueden ser intercambiados o alienados, porque son creados por "reglas fundamentales" que protegen a las personas de riesgos insoportables. 
Un ejemplo sería el derecho a la no discriminación. Si el Homo economicus no sabe qué sexo, color o religión puede tener, independientemente de sus preferencias o sesgos racistas o machistas no estará dispuesto racionalmente a correr el riesgo de ser parte de los discriminados. ${ }^{42}$ Ello es así porque la parte de la libertad a la que renunciaría - la prima del seguro, equivalente a no poder discriminar a otros si se formase parte del grupo favorecido- genera una utilidad marginal menor que la enorme ganancia de utilidad de estar protegido contra la discriminación arbitraria. En consecuencia, el Homo economicus compraría un "seguro completo", es decir, una regla que proteja ese derecho, para maximizar su utilidad esperada.

Podríamos decir lo mismo sobre la educación básica y el derecho a la alimentación, al menos en lo que respecta a la situación en la que no se puede valer por sí mismo (por ejemplo, casos de niños o personas que comienzan sus vidas con discapacidades significativas). Independientemente de las preferencias y juicio sobre la provisión de asistencia social en su sentido más omnicomprensivo, un ser económicamente racional siempre se mostrará racionalmente partidario de algún tipo de seguro completo proporcionado por la sociedad contra esos riesgos, independientemente de los mecanismos que se prefieran para ello. En otras palabras, dicha asistencia puede provenir de alguna forma de Estado, estructuras de clubes o sistemas de benevolencia, y su intensidad puede ser motivo de discrepancia, pero su existencia siempre será preferida a la situación de absoluto desamparo.

Por otro lado, sería mucho más dudoso sostener que un derecho incondicional a la propiedad pueda incluirse aquí. Es evidente que algunos miembros de la sociedad podrían querer racionalmente someter ese derecho a algunas condiciones, como el bienestar colectivo. Es por ello razonable asumir que, en caso de contradicción entre el derecho a la alimentación y el derecho a la propiedad, todo el mundo preferiría asegurarse contra la inanición que protegerse contra la privación de su suntuosa riqueza.

Como adelantábamos más arriba, este conjunto de derechos no se crea por el hecho de ser el Homo economicus un Homo sociabilis, ${ }^{43}$ sino que se establecen en una etapa anterior precisamente para hacer frente al riesgo que surge de la existencia de otros seres humanos frente a los cuales el individuo quiere estar protegido (o por quien el individuo quiere ser ayudado). La forma de protegerse a uno mismo es preestableciendo las reglas bajo las cuales uno aceptaría la coexistencia con los otros elementos de esa sociedad. El establecimiento de derechos inalienables es una decisión egoísta.

\footnotetext{
${ }^{42}$ Nótese que si sería posible aceptar dicho riesgo "irracionalmente".

${ }^{43}$ Con este término se hace referencia a un ser humano que vive en sociedad. No debe confundirse con el Homo sociologicus de P. HiRsCH; S. Michaels; R. Friedman, "«Dirty hands» versus «clean models» - Is sociology in danger of being seduced by economics?", Theory and Society, vol. 16, 3, 1987, p. 322.
} 
Por ello, el razonamiento aquí presentado no deja lugar para el relativismo. Se puede discutir sobre qué derechos inalienables deberían incluirse aquí, ya que esto conlleva un cierto grado de subjetividad, pero no sobre la idea en sí misma de su existencia asumiendo los postulados de la doctrina económica neoclásica. En consecuencia, los derechos inalienables del Homo economicus son per se universales, porque son los mismos para cualquier persona, incluso si sus fundamentos son per se subjetivos.

Finalmente, es necesario reconocer los límites de este ejercicio teórico, en gran medida anunciados ya al comienzo del artículo. En primer lugar, el proceso racional de elección, es decir la subjetividad intrínseca del razonamiento de establecer una preferencia en base a una utilidad marginal superior, requiere que una persona posea las capacidades mentales para realizar dicho proceso de elección. Ello podría entenderse como una limitación de la aplicación de esta teoría económica para aquellos casos de personas con discapacidades mentales. Considero esta crítica falaz.

Que una persona se vea privada de sus capacidades mentales hasta el punto de no poder realizar una elección económicamente racional en absoluto afecta a una construcción que se fundamenta en gran medida en ciertos mecanismos de razonamiento esculpidos en nuestro cerebro. Que un cerebro dado no pueda realizarlos, bien porque no esté suficientemente desarrollado, como en el caso de un menor; o porque sufra de una discapacidad transitoria, una enajenación mental por ejemplo; o bien porque no sea objetivamente capaz de hacerlo, aplicable a casos de discapacidad intelectual severa; en nada afecta a los derechos derivados de dicho ejercicio, que sí cubrirían a esas personas. Las trazas de personalidad que consideramos aquí necesarias para el proceso de elección del Homo economicus se refieren a un ser con un proceso de raciocinio en plena capacidad. Los resultados de dicho proceso, en cambio, son aplicables a todo ser humano, independientemente de sus capacidades.

En segundo lugar, este razonamiento es reduccionista al igualar "ser humano" y "Homo economicus". Debo estar de acuerdo. Como señalaba anteriormente, el ser humano es mucho más que un Homo economicus. En ocasiones podemos actuar de manera absolutamente irracional desde un punto de vista económico, y no por ello estar menos satisfechos con nuestra decisión. En cualquier caso, el Homo economicus, como traza de nuestra personalidad desarrollada de manera evolutiva en nuestra especie nos permite conjeturar que cuando nos enfrentamos a situaciones como las descritas en este trabajo, nuestras decisiones, cualesquiera que sean, serán coherentes con el principio de la utilidad marginal y la maximización del bienestar. Estas elecciones son demasiado importantes para que no sea así. $^{44}$

\footnotetext{
${ }^{44}$ Quiere decirse con ello, que cuando las decisiones implican consecuencias desde el punto de vista evolutivo, como exponerse a la extinción de la especie, no parece arriesgado suponer que nuestra decisión será tomada teniendo en cuenta la utilidad de la
} 
En tercer lugar, puede argumentarse que con estos fundamentos sólo se pueden derivar un número limitado de derechos incluidos como inalienables. Debo estar de acuerdo también. Buscar otros fundamentos para nuestros derechos inalienables, como la dignidad humana, nos conduciría a un catálogo más amplio $-y$ sin duda más justo, desde una perspectiva social- de derechos. Sin embargo, como señalé en la introducción, a diferencia de otros fundamentos más amplios, el razonamiento económico nos permite superar la crítica relativista de una manera más confortable y sólida si el debate se establece en términos de sensibilidad cultural, al evitar justamente el elemento socio-cultural en la construcción de los derechos.

Por último, sirva este artículo como invitación a otros autores a reaccionar a esta línea de razonamiento. En aras de claridad argumentativa, se ofrece un marco para ello, distinguiendo si la reacción se centra en la secuencia del razonamiento, sus conclusiones, los fundamentos de la economía neoclásica, o la posibilidad de que alguien acepte uno de los riesgos presentados.

\section{BIBLIOGRAFÍA}

Asamblea General de las Naciones Unidas, "Declaración Universal de Derechos Humanos", fecha de consulta 23 septiembre 2018, en http://www.un.org/es/universal-declaration-human-rights/.

Aspromourgos, T., "On the origins of the term «neoclassical»", Cambridge Journal of Economics, 10 (3), 265-270, 1986. https://doi.org/10.1093/oxfordjournals.cje.a034999

BECKER, G. S., "Altruism, Egoism, and Genetic Fitness: Economics and Sociobiology", Journal of Economic Literature, 14(3), 817-826, 1976.

BECKER, G. S., "Altruism in the Family and Selfishness in the Market Place", Economica, 48 (189), 1-15, 1981. https://doi.org/10.2307/2552939

Beitz, C. R.; Goodin, R. E., Global Basic Rights, Oxford University Press, New York, 2009. https://doi.org/10.1093/acprof:osobl/9780199604388.001.0001

BEUCHOT, M., Los fundamentos de los derechos humanos en Bartolomé de las Casas, Anthropos Editorial, 1994.

Brown, D. G., "What Is Mill's Principle of Utility?", Canadian Journal of Philosophy, 3(1), $1-12$, 1973. https://doi.org/10.1080/00455091.1973.10716066

misma. Especialmente si una de las opciones, conlleva una utilidad igual a cero como sería la ausencia de utilidad para el propio individuo y para todo otro individuo de su especie. La única salvedad, sería si existiese una persona que creyese que la extinción de la raza humana podría servir a un interés superior. Un ejemplo sería si dicha persona pensase que la aniquilación del ser humano es la única manera de garantizar la supervivencia de la vida en la tierra. Rosenzweig presenta esta idea en su libro de una manera humorística, recordando a Shakespeare, M. L. RoSENZWEIG, Win-win Ecology: How the Earth's Species Can Survive in the Midst of Human Enterprise, Oxford University Press, 2003, pp. 159-160. El mapa de Sanderson et al. es bastante más trágico, E. W. SANDERSON Y OTROS, "The Human Footprint and the Last of the Wild", BioScience, vol. 52, 10, 2002. 
Buchanan, J. M.; Tollison, R. D., The Theory of public choice--II, University of Michigan Press, 1984. https://doi.org/10.3998/mpub.7229

Chander, A.; Chander, A., "Is Nozick Kicking Rawls's Ass - Intellectual Property and Social Justice Symposium: Intellectual Property and Social Justice: Foreword", U.C. Davis Law Review, 40, 2006, pp. 563580.

ColANDER, D., "The Death of Neoclassical Economics", Journal of the History of Economic Thought, 22 (2)(2, 2000, pp. 127-143. https://doi.org/10.4337/9781786435903.00013

Condé, H. V., A Handbook of International Human Rights Terminology, University of Nebraska Press, Lincoln, 2004.

Cruft, R.; LiaO, S. M.; Renzo, M. (eds.), Philosophical Foundations of Human Rights, Oxford University Press, Oxford, New York, 2015.

DonNelLy, J., "The Relative Universality of Human Rights", Human Rights Quarterly, 29(2), 281-306, 2007. https://doi.org/10.1353/hrq.2007.0016

Dworkin, R., "What is Equality? Part 2: Equality of Resources", Philosophy \& Public Affairs, 10(4), 283-345, 1981.

EXECUTIVE BoARd, American Association of ANTHRopology, "Statement on Human Rights", American Anthropologist, 49(4, 1947, pp. 539-543.

FiCHTE, J. G., Grundlage Des Naturrechts Nach Prinzipien Der Wissenschaftslehre, Nabu Press, 2011.

FinemAN, M.; DOUGHERTY, T., Feminism confronts homo economicus: gender, law, and society, Cornell University Press, 2005.

FinNis, J., Natural Law and Natural Rights, Second Edition, Oxford University Press, Oxford, New York, 2011.

HiRsCH, P.; MICHAELS, S.; FRIEDMAN, R., "«Dirty hands» versus «clean models» - Is sociology in danger of being seduced by economics?", Theory and Society, 16(3), 317-336, 1987. https://doi.org/10.1007/bf00139485

Hoffman, M. L., Empathy and Moral Development: Implications for Caring and Justice, Cambridge University Press, 2001.

Jenco, L., "Revisiting Asian Values", Journal of the History of Ideas, 74(2), 237-258, 2013. https://doi.org/10.1353/jhi.2013.0014

KingsbuRY, D., "Universalism and Exceptionalism in "Asia"", en Human Rights in Asia, Springer, 2008. https://doi.org/10.1057/9780230615496_2

MAYA MuÑOZ, G., "La teoría neoclásica: reflexiones.", Ensayos de Economía, 4(7), 163-188, 1993.

MüLlER, C., "The Veil of Uncertainty Unveiled", Constitutional Political Economy, 9(1), 5-17, 1998.

NAgel, E., "Assumptions in Economic Theory", The American Economic Review, 53(2), 211-219, 1963.

Nozick, R., Anarchy, State, and Utopia, Basic Books, 1977.

O'MANique, J., The Origins of Justice: The Evolution of Morality, Human Rights, and Law, University of Pennsylvania Press, 2003. 
O'MANique, J., "Universal and Inalienable Rights: A Search for Foundations", Human Rights Quarterly, 12(4), 465-485, 1990. https://doi.org/10.2307/762495

Orend, B., Human Rights: Concept and Context, Broadview Press, 2002.

Peces-Barba Martínez, G.; Fernández García, E.; De Asís Roig, R.; Fernández Liesa, C. R.; AnsuÁtegui Roig, F. J. (eds.), Historia de los derechos fundamentales, Dykinson, Madrid, 2003.

PERSKY, J., "The ethology of homo economicus", Journal of Economic Perspectives, 9(2),

221-231,

1995.

https://doi.org/10.1257/jep.9.2.221

Posner, R. A., "Rational Choice, Behavioral Economics, and the Law", Stanford Law Review, 50(5), 1551-1575, 1998. https://doi.org/10.2307/1229305

RAWLS, J., A Theory of Justice, Oxford University Press, 1999.

RAWLS, J., "Justice as fairness", The philosophical review, 67(2), 164-194, 1958. Reeditado en Contemporary Political Theory: A Reader, Sage, 13-21, 2004. https://doi.org/10.4135/9781446215272.n2

RAWLS, J., "Reply to Alexander and Musgrave", The Quarterly Journal of Economics, 88(4), 633-655, 1974. https://doi.org/10.2307/1881827

ReAle, M., Teoria tridimensional do direito, Saraiva, São Paulo, 1994.

RENTELN, A. D., International Human Rights: Universalism Versus Relativism, Quid Pro Books, 2013.

DE los Rios URIARTE, M. E., "Las dimensiones trascendentes de la dignidad humana como fundamento para la formulación de los derechos humanos.", Dereito, 23(1), 33-51, 2013.

RosenzWeig, M. L., Win-win Ecology: How the Earth's Species Can Survive in the Midst of Human Enterprise, Oxford University Press, 2003.

Sanderson, E. W.; Jaiteh, M.; LeVy, M. A.; Redford, K. H.; Wannebo, A. V.; Woolmer, G., "The Human Footprint and the Last of the Wild", BioScience, 52(10), 891-904, 2002. https://doi.org/10.1641/00063568(2002)052[0891:thfatl]2.0.c0;2

SCULL, A., "Mind, brain, law and culture", Brain, 130(2, 2007, pp. 585-591.

SEN, A. K., "Democracy as a universal value", Journal of democracy, 10(3), 3-17, 1999. https://doi.org/10.1353/jod.1999.0055

SHeSTACK, J. J., "The Philosophic Foundations of Human Rights", Human Rights Quarterly, 20(2), 201-234, 1998. https://doi.org/10.1353/hrq.1998.0020

SHESTACK, J. J., "The philosophic foundations of human rights", en Human Rights: Concept and Standards, Routledge, Abingdon \& New York, 3168, 2016. https://doi.org/10.4324/9781315183510

ShuE, H., Basic Rights: Subsistence, Affluence, and U.S. Foreign Policy, Princeton University Press, Princeton, 1996.

Stigler, G., "Economics or ethics?", 1980, Harvard University, fecha de consulta 30-11-2018, https://tannerlectures.utah.edu/_documents/a-to-z/s/stigler81.pdf.

ThALER, R. H., "From Homo Economicus to Homo Sapiens", Journal of Economic Perspectives, 14(1), 2000. https://doi.org/10.1257/jep.14.1.133 
Union EuRopea, Carta de los Derechos Fundamentales de la UE, 2010.

VESETH, M., "The Economics of Property Rights and Human Rights", American Journal of Economics and Sociology, 41(2), 169-169, 1982. https://doi.org/10.1111/j.1536-7150.1982.tb03169.x

ZekI, S.; Goodenough, O. (eds.), Law and the Brain, Oxford University Press, Oxford, New York, 2006. 